\title{
La ciencia de animales de laboratorio
}

\author{
Ekaterina Akimovna Botovchenco Rivera, MV, Ph.D.*
}

$\mathrm{T}$

odas las profesiones tienen que acompañar la evolución de la sociedad para que puedan mantenerse activas y contar con la credibilidad de esa misma sociedad. Con la Medicina Veterinaria no es diferente, y para poder hacer frente a las nuevas demandas de los avances de la ciencia y de la biotecnología, derribando conceptos históricos e introduciendo otros nuevos, fue necesario vislumbrar nuevas áreas dentro de su historia. Aún más, tuvo que pasar de una profesión puramente técnica a una profesión en que la ética guíe su caminar. Una profesión que trate del bienestar de seres vivos, los cuales están en el centro de la actuación del hombre generando sentimientos de preocupación y de respeto. Por eso, la Medicina Veterinaria tiene que dar a conocer a esa sociedad su modo ético de actuar y demostrar que ayudando a los animales estará también buscando el bienestar del hombre.

Dentro de ese contexto de cambios en la Medicina Veterinaria, una de las áreas que se destaca por su preocupación con la ética en el uso de los animales es la de la Ciencia de Animales de Laboratorio. Esta área se encuentra en una fase de rápido desarrollo y, aunque los animales hayan sido objetos de experimentación desde el principio de la humanidad, como disciplina estructurada aún es bastante nueva.

Esa ciencia es muchas veces atacada por fuerza de presiones emocionales, políticas y percepción equivocada de algunas personas. Sin embargo, se preocupa por el bienestar y uso ético de animales desde hace más de dos siglos, incluso buscando reglamentarla bajo leyes, siendo una de las primeras en Inglaterra el 1876 Cruelty to Animal's Act, que ya preconizaba: "reconsiderar las necesidades de la ciencia con las necesidades humanitarias de los animales".

Pero antes de que existieran esas leyes, dos científicos ingleses, Marshall y Hall, en 1831, enunciaron principios éticos para el uso de animales en experimentación, los cuales, aunque no tuvieron el impacto deseado en la época, sirvieron de inspiración para que otros principios fueran desarrollados más adelante. El más importante fue el de las tres R, enunciado por Russell y Burch en 1959 (Replacement, Reduction, Refinement), base de las legislaciones en todo el mundo y que ha cambiado la cara del uso de animales en experimentación, enseñanza y pruebas.

¿Y qué nos dicen esas tres R? Que nunca utilicemos animales si se pueden usar alternativas, siempre usar un mínimo necesario de animales y refinar los procedimientos de crianza, cuidado y uso para minimizar el sufrimiento causado y la promoción de investigaciones relevantes a las tres $\mathrm{R}$. La aplicación de esos principios trajo la reducción en el uso de animales, grandes ganchos en el bienestar de estos y rápidos avances en las ciencias biomédicas.

Pero necesitamos que los principios de la ética surjan dentro de uno naturalmende la Universidad Federal de Goiás, Goiás, Brasil te, y se concreten en actitudes éticas como una extensión de nuestros pensamientos. La creación de comités de ética en el uso de animales, sean esos conocidos como IACUCs, 
CEUAS, CICUALES, etcétera, todos con la misma finalidad, han sido esenciales para la implementación de esos principios éticos, tornando lo que era un concepto abstracto en realidad aplicada a las prácticas del día a día.

El camino es largo y en constante evolución, así que debemos siempre acordarnos que usar animales en investigación es un privilegio que la sociedad brinda a la comunidad científica con la expectativa de que tal uso proveerá nuevos conocimientos o un estado de mejor bienestar de hombres y de animales.

La Medicina Veterinaria, como profesión preocupada por el bienestar animal, tiene el papel de concientizar a todos los que utilizan animales en experimentación, enseñanza o pruebas para los cuidados y manejos que deben ser proporcionados a esos seres vivos, a fin de que no sufran dolor, angustia, miedo o ansiedad. Y para que podamos ayudar a los animales a mantener su bienestar es necesario conocer la biología y etología de la especie animal. Ese es el conocimiento con el cual vamos trabajar, y eso se consigue por medio de cursos y de mucha revisión de literatura.

Por eso celebramos la idea de la revista Spei Domus de dedicar un número con artículos específicos del área de la Ciencia de Animales de Laboratorio, llenando una laguna que hay en esa área. 